\title{
BIOTURBATION RATES IN BAHÍA LA CHOYA, SONORA, MEXICO
}

\section{RAZONES DE BIOTURBACIÓN EN BAHÍA LA CHOYA, SONORA, MÉXICO}

\author{
J. Lynn Myrick* \\ Karl W. Flessa* \\ Department of Geosciences \\ University of Arizona \\ Tucson, AZ 85721 \\ USA
}

Recibido en abril de 1995; aceptado en noviembre de 1995

\begin{abstract}
Experimental determination of sediment reworking rates in a subtropical intertidal flat environment yielded information about the amount, nature and implications of sediment reworking in nearshore deposits. Callianassid shrimp in Bahía La Choya, Sonora, Mexico, overturn the sediment in the inner flats at an average rate of $0.56 \mathrm{~m}^{3} / \mathrm{m}^{2} /$ year. Elasmobranch rays overturn the sediment in the midflats at an average rate of $1.01 \mathrm{~m}^{3} / \mathrm{m}^{2} /$ year. Resin castings indicate that the shrimp are capable of burrowing to a depth of at least $1.15 \mathrm{~m}$ and, where present, can completely rework this interval in Bahía La Choya in two years. The rays reach a maximum observed burrowing depth of $20 \mathrm{~cm}$ and, where present, can completely rework this interval in Bahia La Choya in 72 days. Reworking rates are high enough to preclude the preservation of most physical sedimentary structures under normal conditions. Only large-scale sedimentary structures or those buried deeply and rapidly are likely to cscape reworking. Rates of biogenic sedimentation by callianassid shrimp are high enough to generate subsurface shell beds. Short-term biogenic sedimentation rates are higher than long-term rates, indicating that such intertidal sediments are not only thoroughly reworked, but are incomplete at time scales of weeks to months.
\end{abstract}

Key words: bioturbation, sediment reworking, callianassid shrimp, elasmobranch rays.

\section{RESUMEN}

La determinación experimental de las razones de retrabajamicnto en un ambiente de planicie de marea subtropical proporcionó información acerca de la cantidad, naturaleza e implicaciones del retrabajamiento de los sedimentos en depósitos próximos a la costa. Los camarones calianásidos en bahía La Choya, Sonora, México, revuelven el sedimento en las planicies interiores a una razón promedio de $0.56 \mathrm{~m}^{3} / \mathrm{m}^{2} /$ año. Las rayas revuelven el sedimento en las planicies medias a una razón promedio de $1.01 \mathrm{~m}^{3} / \mathrm{m}^{2} / a n ̃ o$. Los moldes de resina indican que el camarón es capaz de enterrarse a una profundidad de al menos $1.15 \mathrm{~m}$ y, donde se presenta, puede completamente retrabajar este intervalo en bahía La Choya en dos años. Las rayas alcanzan una profundidad de enterramiento máxima observable de $20 \mathrm{~cm}$ y, donde se presentan, pueden completamente retrabajar este intervalo en bahía La Choya en 72 días. Las razones de retrabajamiento son lo suficientemente altas como para evitar, bajo condiciones normales, la preservación de la mayoria de las estructuras sedimentarias. Es probable que sólo las estructuras sedimentarias de gran escala, o aquellas enterradas profunda y rápidamente, escapen el

- Present address: Brown and Root Environmental, 900 Trail Ridge Rd., Aitken, SC 29803, USA.

* Please address all correspondence and reprint requests to Flessa. 
retrabajamiento. Las razones de sedimentación biogénica por camarones calianásidos son lo suficientementc altas como para generar lechos de conchas subsuperficiales. La sedimentación biogénica a corto plazo es mayor que la de a largo plazo; esto indica que los sedimentos intermareales no sólo están totalmente retrabajados, sino que están incompletos a escalas de tiempo de semanas a meses.

Palabras clave: bioturbación, retrabajamiento de sedimentos, camarones calianásidos, rayas.

\section{INTRODUCTION}

Ironically, burrowing organisms, while providing invaluable information in paleobiological studies, often cause difficulties in other types of studies. Trace fossils provide information on the behavior and life habits of organisms at the expense of information on the physical aspects of the environment of deposition provided by physical sedimentary structures. However, trace fossils can contribute to sedimentological information, such as the degree of reworking of the sediment by animals. Visual techniques, such as ichnofabric index analysis (e.g., Droser and Bottjer, 1986; Taylor and Goldring, 1993), are often used to estimate the relative magnitude of reworking, but the rate at which reworking occurs has proved more difficult to measure.

Most attempts to estimate rates of bioturbation have focused on modern environments, where sediment reworking by organisms can be observed and measured. Such actualistic studies have proven useful in interpreting ancient deposits and their sedimentary structures. The consequences of bioturbation include the destruction of primary physical sedimentary structures, perhaps within a short time. For example, inactive sand ripples in the US Virgin Islands were destroyed within two to four weeks due to bioturbation by fishes, though the traces made by the fishes remained intact for several months (Clifton and Hunter, 1973). Meyers (1977a, b) established summer overturn rates for a coastal lagoon dominated by holothurians and polychaetes. The top $10 \mathrm{~cm}$ of sediment was reworked in 0.5 to 2.4 years, removing primary physical sedimentary structures in the process.

This study considers two bioturbating organisms found in a modern tidal flat environment: callianassid shrimp and elasmobranch rays. The structures created by callianassid

\section{INTRODUCCIÓN}

Irónicamente, mientras los organismos enterradores proveen valiosa información en estudios paleobiológicos, también con frecuencia causan dificultades en otros tipos de estudios. Los icnofósiles proveen información sobre el comportamiento y hábitos de vida de los organismos, a costa de la información de los aspectos fisicos del ambiente de depositación que proveen las estructuras sedimentarias. Sin embargo, los icnofósiles pueden contribuir a la información sedimentológica, como el grado de retrabajamiento del sedimento por animales. Técnicas visuales, tales como el análisis del índice de la icnofábrica (e.g., Droser y Bottjer, 1986; Taylor y Goldring, 1993), son usadas con frecuencia para estimar la magnitud relativa de retrabajamiento, pero la razón a que ocurre tal retrabajamiento ha resultado ser más difícil de medir.

La mayoria de los intentos para estimar las razones de bioturbación se han enfocado en ambientes modernos, donde el retrabajamiento de los sedimentos por organismos puede ser observado y medido. Tales estudios "actualísticos" han resultado ser útiles para interpretar depósitos antiguos y sus estructuras sedimentarias. Las consecuencias de la bioturbación incluyen la destrucción de las estructuras sedimentarias primarias, quizás en un corto periodo de tiempo. Por ejemplo, rizaduras de arena inactivas en las Islas Vírgenes fueron destruidas entre dos y cuatro semanas por la bioturbación debida a peces, aunque las huellas hechas por los peces permanecieron intactas por varios meses (Clifton y Hunter, 1973). Meyers (1977a. b) establecieron razones de revolvimiento en verano para una laguna costera dominada por holoturias y poliquetos. Los $10 \mathrm{~cm}$ superiores de sedimento fueron retrabajados en 0.5 a 2.4 años, destruyendo las estructuras sedimentarias en el proceso. 
shrimp and elasmobranch rays are both conspicuous and abundant in a subtropical tidal flat in Bahía La Choya, Sonora, Mexico. How effective are these organisms at reworking the sediment and how would this reworking affect the resulting sedimentary record of the tidal flats?

\section{SEDIMENT REWORKING BY CALLIANASSID SHRIMP AND ELASMOBRANCH RAYS}

\section{Callianassid shrimp}

Callianassid shrimp are known to be deep burrowers and have received much attention in the literature (e.g., Pemberton et al., 1976; Ott et al., 1976; Suchanek, 1983; Miller and Myrick, 1992). Suchanek (1983) demonstrated that seagrass communities can be strongly controlled and adversely affected by the presence of callianassids, as can neighboring animal communities (Aller and Dodge, 1974; Tamaki, 1988; Posey, 1986).

The effects on sediments by burrowing shrimp have been extensively investigated (Vaugelas, 1984). The volume and depth of oxidized sediments have been shown to increase up to sixteen times due to such activity (Ott $e t$ al., 1976). Sediment compaction can also be reduced by callianassid burrowing (Ronan et al., 1981). Sediment size distribution appears to both control and be controlled by the presence of callianassid burrows. Griffis and Chavez (1988) indicated that sediment grain size distribution may determine the size and distribution of callianassid burrows. In Mugu Lagoon, California, sediment homogenization has occurred where shrimp populations are high (Miller and Myrick, 1992; Warme, 1967).

The depth of shrimp burrows varies with species and possibly with sediment type. Burrows of Axius serratus reach to more than $2 \mathrm{~m}$ as shown hy resin castings (Pemherton ot al, 1976; technique modified from Shinn, 1968). Callianassid shrimp generally do not reach these depths, but most species are thought to burrow to a depth of $50 \mathrm{~cm}$ or more (Miller and Myrick, 1992; Miller et al., 1990).
Puesto que las estructuras creadas por camarones calianásidos y rayas son conspicuas y abundantes en las planicies de marea subtropical en bahía La Choya, Sonora, México, en el presente estudio se consideran estos dos organismos bioturbadores para determinar qué tan efectivos son en retrabajar el sedimento y cómo este retrabajamiento afecta el registro sedimentario resultante en las planicies de mareas.

\section{RETRABAJAMIENTO DEL SEDIMENTO POR CAMARONES CALIANÁSIDOS Y RAYAS}

\section{Camarones calianásidos}

Los camarones calianásidos son conocidos por ser enterradores profundos $y$ han recibido mucha atención en la literatura (e.g., Pemberton et al., 1976; Ott et al., 1976; Suchanek, 1983; Miller y Myrick, 1992). Suchanek (1983) demostró que las comunidades de pastos marinos pueden ser fuertemente controladas y adversamente afectadas por la presencia de calianásidos, así como también lo pueden ser las comunidades vecinas (Aller y Dodge, 1974; Tamaki, 1988; Posey, 1986).

Los efectos sobre los sedimentos por el camarón enterrador han sido extensamente investigados (Vaugelas, 1984). Se ha encontrado que el volumen y la profundidad de los sedimentos oxidados se incrementan hasta dieciséis veces debido a tal actividad (Ott et al, 1976). La compactación del sedimento también puede ser disminuida por el enterramiento del calianásido (Ronan et al, 1981). La distribución de tamaño del sedimento parece controlar y parece ser controlada por la presencia de madrigueras de calianásidos. Griffis y Chávez (1988) indicaron que la distribución de tamaño de grano del sedimento puede determinar el tamaño y distribución de las madrigueras de los calianásidos. En la laguna Mugu, California, la homogeneización del sedimento ha ncurrido donde las poblaciones de camarones son altas (Miller y Myrick, 1992; Warme, 1967).

La profundidad de las madrigueras de los camarones varía con la especie y posiblemente con el tipo de sedimento. Las madrigueras de 
The first quantitative estimate of sediment reworking due to the activities of callianassids was MacGinitie and MacGinitie's (1949) estimate of 20 to $50 \mathrm{~cm}^{3} /$ day for each burrow in Mugu Lagoon, California. Swinbanks and Luternauer (1987) estimated that, in the Fraser Delta (British Columbia) tidal flat, overturn due to Callianassa californiensis is $24 \pm 12 \mathrm{~g}$ of sediment per shrimp per day. Shrimp population density in Mugu Lagoon fluctuated appreciably within a short time period (Miller and Myrick, 1992), so total rates of reworking may vary through time. Since the amount of reworking is most closely related to the volume and abundance of structures, estimates of overturn must account for the density of structures in the area being studied and should therefore be expressed in terms of a volume per area with respect to time.

Deep bioturbation creates several stratigraphic effects. The burrows themselves may be preserved and can serve as excellent paleoenvironmental indicators (see Bromley, 1990). In addition, hioturbation by callianassids has been invoked as a mechanism for biogenic stratification (Meldahl, 1987) - the formation of subsurface shell beds (fig. 1). Such shell beds can cause "taphonomic inhibition" of subsequent burrowing (Miller et al., 1990). Graded bedding can also be created by burrowing callianassids (Warme, 1971; Tudhope and Scoffin, 1984). Trace metals can concentrate in the burrow walls of Callianassa major (Over, 1990), while some workers have shown that bioturbation, in general, can be a factor in increasing contaminant transport within sediments (Bosworth and Thibodeaux, 1990)

\section{Elasmobranch rays}

Although ray burrows, or pits, have been less studied than callianassid burrows, modern ray feeding pits have been recognized since at least 1935 (MacGinitie, 1935). Howard et al. (1977) considered them recognizable in the fossil record. Modern structures are widespread geographically; they have been found on both coasts of North America (Warme, 1971; Cook, 1971; MacGinitie, 1935; Grant, 1983),
Axius serratus alcanzan más de $2 \mathrm{~m}$, como se ha demostrado mediante moldes de resina (Pemberton et al., 1976; técnica modificada de Shinn, 1968). Los camarones calianásidos generalmente no alcanzan estas profundidades, pero se piensa que se entierran a una profundidad de $50 \mathrm{~cm}$ o más (Miller y Myrick, 1992; Miller et al., 1990).

La primera estimación cuantitativa de retrahajamiento de sedimentos debido a las actividades de calianásidos fue la de MacGinitie y MacGinitie (1949), de 20 a $50 \mathrm{~cm}^{3} /$ dia por cada madriguera en la laguna Mugu, California. Swinbanks y Luternauer (1987) estimaron que, en la planicie de marea deltaica del Fraser (Colombia Británica), el revolvimiento debido a Callianassa californiensis es de $24 \pm 12 \mathrm{~g}$ de sedimento por camarón por día. La densidad de la población de camarón en la laguna Mugu parece fluctuar apreciablemente dentro de cortos periodos de tiempo (Miller y Myrick, 1992), de tal forma que las razones totales de retrabajamiento pueden variar a través del tiempo. Puesto que la cantidad de retrabajamiento está más estrechamente relacionada con el volumen y abundancia de estructuras, las estimaciones de revolvimiento deben tomar en cuenta la densidad de estructuras en el área de estudio y, por lo tanto, se deben expresar en términos de un volumen por área con respecto al tiempo.

La bioturbación profunda origina varios efectos estratigráficos. Las madrigueras mismas pueden ser preservadas y servir como excelentes indicadores paleoambientales (ver Bromley, 1990). Adicionalmente, se ha utilizado la bioturbación por calianásidos como un mecanismo de estratificación biogénica (Meldahl, 1987) en la formación de capas de conchas subsuperficiales (fig. 1). Tales capas de conchas pueden causar una "inhibición tafonómica" para enterramientos subsecuentes (Miller et al., 1990). La estratificación graduada también puede ser causada por calianásidos enterradores (Warme, 1971; Tudhope y Scoffin, 1984). Los metales traza pueden concentrarse en las paredes de las madrigueras de Callianassa major (Over, 1990), mientras que algunos autores han mostrado que, en general, la bioturbación puede ser un factor que incrementa el transporte 


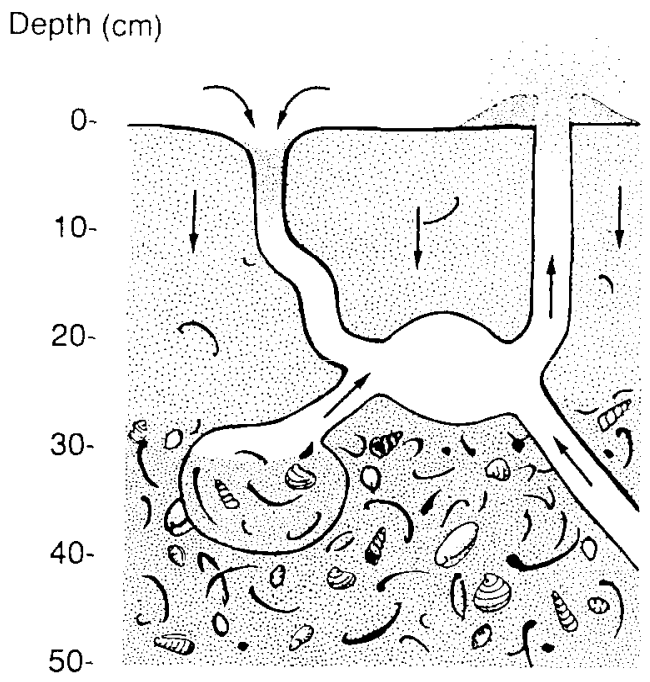

Figure 1. Callianassid burrows and the biogenic stratification of shell material they produce. Adapted from Meldahl (1987).

Figura 1. Madrigueras de los calianásidos y la estratificación biogénica de las conchas que producen. Adaptado de Meldahl (1987).

Australia (Whitky, 1940; Bigelow and Schroeder, 1953), and New Zealand (Gregory et al., 1979). Bioturbation by other fishes has been recognized both in modern environments (Risk and Craig, 1976; Stanley, 1971) and in the fossil record (Dubiel et al., 1987; McAllister, 1988; Anderson, 1976).

Nichols (1965) first reported ray excavations in the Gulf of California and Fürsich et al. (1991) documented ray feeding excavations throughout the flats of Bahía La Choya. They suggested that these structures could represent significant amounts of sediment reworking and could therefore contribute to the destruction of other sedimentary structures.

Ray pit morphology generally consists of an ellipsoidal or circular dish-shaped pit with a deeper excavation in the middle (fig. 2). Howard et al. (1977) proposed a simple "wingflapping" model for the origin of the pit, in which the ray creates the excavation by flapping its wings over the sediment surface. Rays are known to cover themselves with sand in this way, especially during mating (Nordell, 1990). de contaminantes dentro de los sedimentos (Bosworth y Thibodeaux, 1990).

\section{Rayas}

No obstante que las excavaciones, u hoyos, de rayas han sido menos estudiadas que las madrigueras de los calianásidos, se han reconocido los hoyos alimenticios de las rayas desde al menos 1935 (MacGinitie, 1935). Howard et al. (1977) las consideran reconocibles en el registro fósil. Las estructuras modernas están ampliamente distribuidas geográficamente; se han encontrado en ambas costas de Norteamérica (Warme, 1971; Cook, 1971; MacGinitie, 1935; Grant, 1983), Australia (Whitky, 1940; Bigelow y Schroeder, 1953), y Nueva Zelanda (Gregory et al., 1979). La bioturbación por otros peces ha sido reconocida tanto en ambientes modernos (Risk y Craig, 1976; Stanley, 1971) como en el registro fósil (Dubiel et al, 1987; McAllister, 1988; Anderson, 1976).

El primer reporte de excavaciones de rayas en el golfo de California fue el de Nichols 


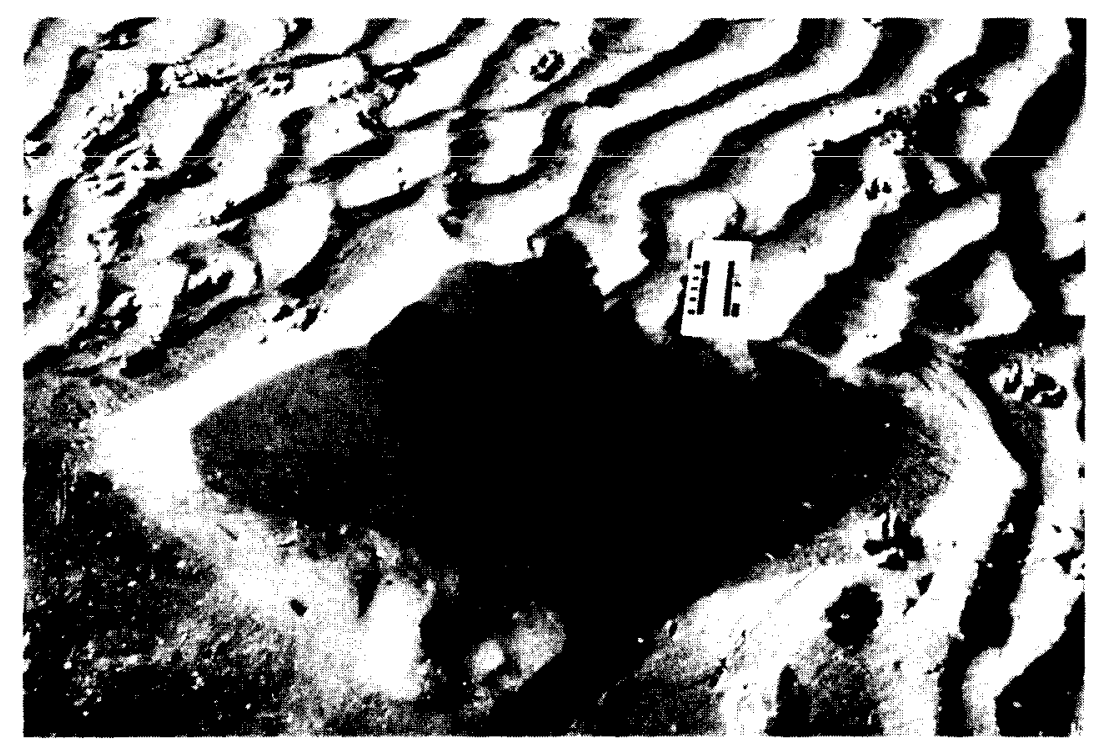

Figure 2. Fresh ray pit from mid flat area of Bahia La Choya. The body outline is preserved, indicating that this pit was created by a bat ray. Solid bar on scale equals $10 \mathrm{~cm}$. The deep portion of the pit was formed directly below the ray's gill clefts, and is already partially filled in here.

Figura 2. Hoyo de raya reciente en las planicies medias de bahía La Choya. Se preservó el perfil del cuerpo, lo cual indica que fue hecho por una raya murciélago. La línea sólida en la escala es igual a $10 \mathrm{~cm}$. La parte profunda del hoyo se formó directamente abajo de las hendiduras branquiales; ya está parcialmente rellenada.

This technique, however, does not account for the deeper part of the pit. Gregory et al. (1979) established the mechanism necessary for producing the deeper section of the pit. They showed that the rays employ hydraulic jetting by forcing water through their gill clefts into the sediment, thus effectively uncovering infaunal animals to a maximum depth of approximately $30 \mathrm{~cm}$.

Grant (1983) addressed ray pits quantitatively as a source of biogenic sediment reworking. Through censuses of the areal extent of ray pits on the surface, he concluded that the reworking of sediment by rays was relatively unimportant $(<1 \%$ of total areal reworking,
(1965), y Fürsich et al. (1991) documentaron las excavaciones alimenticias de rayas a to largo de las planicies de bahía La Choya. Ellos sugirieron que estas estructuras pueden representar cantidades significativas de retrabajo de sedimento $\mathrm{y}$, por tanto, pueden contribuir a la destrucción de otras estructuras sedimentarias.

La morfología de los hoyos de rayas consiste de una excavación de forma elipsoidal o un plato circular con una excavación más profunda en medio (fig. 2). Howard et al. (1977) propusieron un modelo simple de "ala ondeando" para el origen del hoyo, en el cual la raya crea la excavación por el movimiento ondulatorio de sus aletas sobre la superficie del 
$1.4-2.7 \mathrm{~cm}^{2} / \mathrm{m}^{2}$ ) when compared to that of bedform migration. However, this estimate is based on areal, not volumetric, estimates of sediments disturbed by ray pits. Thrush et al. (1991) determined that sediment in a $700-800 \mathrm{~m}^{2}$ area would be overturned by rays within 70 days in an area of high ray pit density. In addition, they showed that the presence of ray pits affects distribution patterns of infaunal animals during recolonization of pits by infaunal animals.

Clifton and Hunter (1973) attempted to determine how quickly sedimentary structures would be destroyed by biogenic activities which caused lateral shifting of the sediment, including the activity of rays. Their final estimate of the sediment reworking rate was $126 \mathrm{~g} / \mathrm{m}^{2}$ per day. However, like Grant (1983), Clifton and Hunter's technique resulted in an areal estimate of reworking, not a volumetric estimate of sediment reworking.

Gregory (1991) described a Miocene trace fossil that resembled Recent ray feeding structures from New Zealand. Piscichnus waitemata is interpreted as the fossil version of eagle ray feeding pits observed in nearby modern environments. Other examples of this trace fossil predating the ichnological classification include Miller's (1979) "large vertical structures" and Hunter et al.'s (1984) "surf-zone potholes", as well as structures described as ray feeding pits or excavations (Howard et al., 1977; Kamola, 1984; Gregory et al., 1979; Gregory et al., 1983; Gregory, 1981, 1986; Brook, 1983), but not classified further. While some of these strongly resemble modern ray pits, others have only cursory resemblances. Those structures with strong resemblances to modern pits date back to at least the Cretaceous (Howard et al, 1977), while others date back to the Devonian (Miller, 1979).

Ray pits are distinguishable from other fish-produced (Piscichnus) structures, such as those present in the Plio-Pleistocene Koobi Fora Formation (Feibel, 1987), because of the deeper conical hole inside the main structure (Howard et al., 1977; Gregory et al., 1979). Some trench-like feeding depressions have also been attributed to rays (MacGinitie, 1935). sedimento. Se sabe que las rayas se cubren de sedimento en esta forma, especialmente durante el apareamiento (Nordell, 1990). Sin embargo, esta técnica no explica la parte más profunda del hoyo. Gregory et al. (1979) establecieron el mecanismo necesario para producir la sección más profunda del hoyo. Ellos mostraron que las rayas emplean chorros hidráulicos; forzan agua dentro del sedimento a través de sus hendiduras branquiales $\mathrm{y}$, de esta manera, efectivamente descubren animales infaunales hasta una profundidad de $30 \mathrm{~cm}$.

Grant (1983) señaló cuantitativamente los hoyos de rayas como una fuente biogénica de retrabajamiento del sedimento. A través de censos de la extensión por área de los hoyos de raya en la superficie, concluyó que el retrabajamiento del sedimento por las rayas fue poco importante $(<1 \%$ del área total retrabajada, $1.4-2.7 \mathrm{~cm}^{2} / \mathrm{m}^{2}$ ) comparado con la migración de las formas del lecho. Sin embargo, hay que notar que esta estimación está basada en estimaciones por área, no volumétricas, de los sedimentos perturbados por los hoyos de rayas. Thrush et al. (1991) determinaron que el sedimento en un área de $700 \mathrm{a} 800 \mathrm{~m}^{2}$ sería revuelto por las rayas dentro de 70 días en una área de alta densidad de hoyos de rayas; adicionalmente, mostraron que la presencia de hoyos de rayas afecta los patrones de distribución de animales infaunales durante la recolonización de los hoyos por animales infaunales.

Clifton y Hunter (1973) intentaron determinar qué tan rápido serían destruidas las estructuras sedimentarias por las actividades biogénicas que causan el cambio lateral de los sedimentos, incluyendo la actividad de las rayas. Su estimación final de la razón de retrabajamiento del sedimento fue de $126 \mathrm{~g} / \mathrm{m}^{2}$ por día. Sin embargo, al igual que Grant (1983), la técnica de Clifton y Hunter tuvo como resultado una estimación del área y no del volumen de sedimento retrabajado por las rayas.

Gregory (1991) describió un icnofósil del Mioceno que se asemeja a las estructuras de alimentación recientes de las rayas en Nueva Zelanda. Piscichnus waitemata es interpretado como la versión fósil de un hoyo de alimenta- 


\section{STUDY AREA}

Bahia La Choya is located in the northern Gulf of California, just north of the town of Puerto Peñasco (fig. 3). Sedimentation rates in this area are low, because the local rivers are ephemeral and the Colorado River has been extensively dammed, diverting its water and sediment load (Fürsich and Flessa, 1987). Radiocarbon dates on shells also demonstrate low sedimentation rates (Flessa et al., 1993).

The geology of the Puerto Peñasco area consists of late Pleistocene sedimentary rocks overlying plutonic and volcanic rocks. These sedimentary rocks are of mixed carbonateclastic composition. Carbonates are derived from shell fragments, while the clastics are predominantly silicates derived locally from basaltic and granitic sources (for further information see Ekdale, 1987).

Bahía La Choya is a large intertidal complex, consisting of sandflats, rocky flats, marsh, and channels. Covering about $10 \mathrm{~km}^{2}$, it has an unusually high tidal range of up to $8 \mathrm{~m}$ during spring tides. Based on location and sedimentary structures, the flats can be divided into three parts: 1) inner flats, dominated closest to the beach by callianassid shrimp burrows; 2) midflats, dominated by ripples and ray feeding traces; 3) outer flats, with sand waves and occasional biogenic structures (fig. 4). Fürsich et al. (1991) summarize the sediments and sedimentary structures present throughout Bahía La Choya. Bahía La Choya's low sedimentation rate suggests that bioturbation is the dominant process causing burial of shell material, possibly resulting in time-averaging (Flessa et al., 1993) and stratigraphic disorder of fossil sequences (Cutler and Flessa, 1990), as well as concentration of shell material at depth (Meldahl, 1987).

Though many animals contribute to the bioturbation of sediment in Bahía La Choya, this study concentrates on the effects of two of the most conspicuous tracemakers: callianassid shrimp and elasmobranch rays. The shrimp create three-dimensional burrow networks (fig. 1). The rays produce dish-shaped depressions in the sediment that occasionally ción de la raya águila de los ambientes modernos aledaños. Otros ejemplos de este icnofósil predatando la clasificación icnológica incluyen a las "grandes estructuras verticales" de Miller (1979) y "baches de la zona de surf" de Hunter et al. (1984), asi como las estructuras descritas como hoyos de alimentación de rayas o excavaciones sin una clasificación posterior (Howard et al., 1977; Kamola, 1984; Gregory et al., 1979; Gregory et al., 1983; Gregory, 1981, 1986; Brook, 1983). Algunas de éstas se asemejan fuertemente a los hoyos de rayas modernos, mientras que otras tienen sólo una semejanza superficial. Aquellas estructuras con una fuerte semejanza a los hoyos de rayas modernos se remontan al menos hasta el Cretácico (Howard et al., 1977), mientras que otras al Devónico (Miller, 1979).

Los hoyos de rayas se distinguen de otras estructuras producidas por peces (Piscichnus), tales como aquellas presentes en la formación Koobi Fora del Plio-Pleistoceno (Feibel, 1987), por el hoyo cónico más profundo en el interior de la estructura principal (Howard et al., 1977; Gregory et al., 1979). Algunas depresiones de alimentación en forma de trinchera también se han atribuido a rayas (MacGinitie, 1935).

\section{ÁREA DE ESTUDIO}

Bahía La Choya se localiza en el norte del golfo de California, justamente al norte del poblado de Puerto Peñasco (fig. 3). Las razones de sedimentación en el área son bajas, debido a que los ríos locales son efímeros y el extenso número de presas en el río Colorado ha desviado sus aguas y carga de sedimento (Fürsich y Flessa, 1987). El fechado de conchas por radiocarbón también demuestra la baja razón de sedimentación (Flessa et al., 1993).

La geología en el área de Puerto Peñasco consiste de rocas sedimentarias del Pleistoceno tardío sobreyaciendo rocas plutónicas y volcánicas. La composición de las rocas sedimentarias es una mezcla de carbonatos y clásticos. Los carbonatos derivan de fragmentos de conchas, mientras que los clásticos son predominantemente silicatos derivados localmente de fuentes graníticas y basálticas (para información adicional ver Ekdale, 1987). 


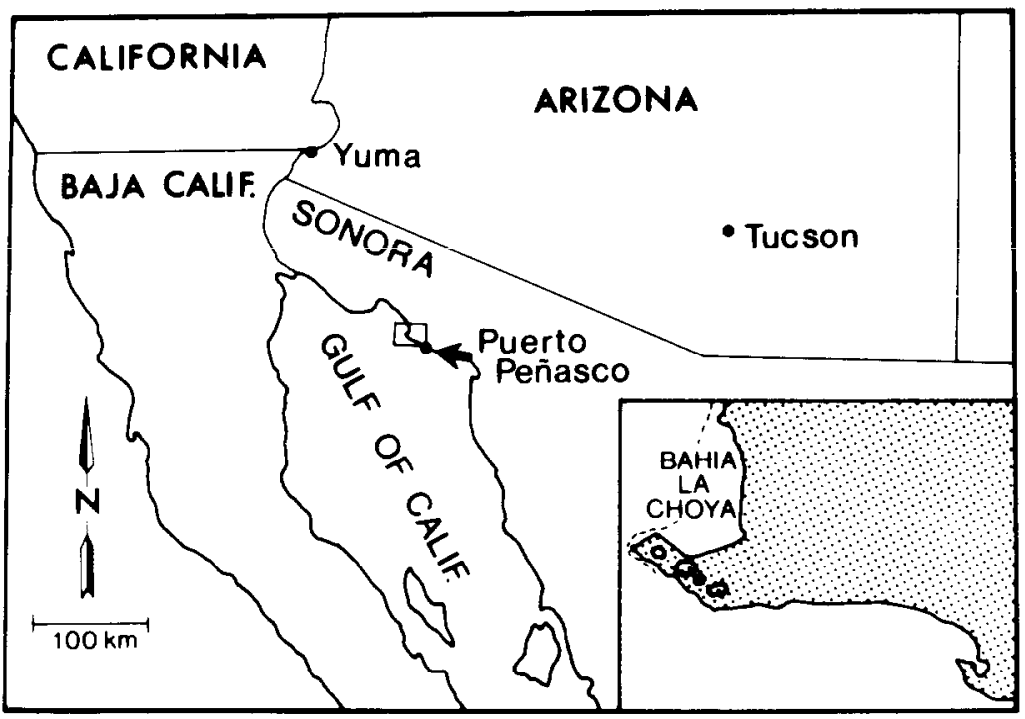

Figure 3. Location map of Bahía La Choya.

Figura 3. Localización de bahía La Choya.

preserve an outline of the ray (fig. 2). The species probably responsible for these traces in Bahía La Choya are the ghost shrimp Callianassa californiensis and the elasmobranch rays Urolophus halleri and Myliobatis californica (fig. 5). In addition, the shovelnose guitarfish Rhinobatos productus is present in Bahía La Choya and may contribute to the production of feeding pits. All of these species were observed in Bahia La Choya during this study.

\section{METHODS}

Sediment overturn by callianassid shrimp was tracked by means of colored shells placed on the surface of the sediment in both the callianassid zone of the inner flats and in a callianassid-free zone of the inner flats. To determine the maximum depth of callianassid burrowing in this area, resin casts were poured in the callianassid zone. Average burrow density was determined by counting burrow openings per square meter. The time and duration of studies are shown in table 1.
Bahía La Choya es un gran complejo intermareal, que consiste de planicies arenosas, planicies rocosas, marismas y canales. Cubre un área de $10 \mathrm{~km}^{2}$ y usualmente tiene un alto intervalo de mareas, de hasta $8 \mathrm{~m}$ durante la primavera. Con base en su localización y las estructuras sedimentarias asociadas, se pueden dividir las planicies en tres partes: 1) planicies internas, dominadas próximas a la playa por madrigueras de camarones calianásidos; 2) planicies medias, dominadas por rizaduras $y$ huellas de alimentación de rayas; 3) planicies externas, con ondas de arena y estructuras biogénicas ocasionales (fig. 4). Fürsich et al. (1991) resumen los sedimentos y estructuras sedimentarias presentes a lo largo de la bahía La Choya. La baja razón de sedimentación en bahía La Choya sugiere que la bioturbación es el proceso dominante que causa el enterramiento de las conchas, posiblemente causando un promediado en el tiempo (Flessa et al., 1993) y el desorden estratigráfico de las secuencias de fósiles (Cutler y Flessa, 1990), asi como la concentración de conchas a profundidad (Meldahl, 1987). 
Table 1. Results of shell burial experiments. Code for seasons: $\mathrm{Sp}=$ spring, $\mathrm{Su}=$ summer. $\mathrm{F}=$ fall. $\mathrm{W}=$ winter. Maximum rate estimates are based on depth of recovcred shclls only; minimum rate cstimates are based on depth of recovered shells, with unrecovered shells assigned a depth of zero.

Tabla 1. Resultados de los experimentos de enterramiento de conchas. Clave de las estaciones: $\mathrm{Sp}=$ primavera, $\mathrm{Su}=$ verano, $\mathrm{F}=$ otoño, $\mathrm{W}=$ invierno. Las mayores razones estimadas se basaron sólo en la profundidad de las conchas recuperadas; las menores razones estimadas se basaron en la profundidad de las conchas recuperadas, con una profundidad igual a cero para las conchas no recuperadas.

\begin{tabular}{ccclcrr}
\hline Site No. & $\begin{array}{c}\text { Start date } \\
(\mathrm{m} / \mathrm{d} / \mathrm{yr})\end{array}$ & $\begin{array}{c}\text { End date } \\
(\mathrm{m} / \mathrm{d} / \mathrm{yr})\end{array}$ & Seasons & Duration & $\begin{array}{c}\text { Max. rate } \\
(\mathrm{cm} / \mathrm{yr})\end{array}$ & $\begin{array}{r}\text { Min. rate } \\
(\mathrm{cm} / \mathrm{yr})\end{array}$ \\
\hline \multicolumn{2}{c}{ Callianassa zone } & & & & & \\
1 & $03 / 16 / 92$ & $11 / 07 / 92$ & Sp-Su-F & 8 months & 17.61 & 8.61 \\
2 & $03 / 16 / 92$ & $02 / 06 / 93$ & Sp-Su-F-W & 11 months & 5.54 & 4.32 \\
3 & $09 / 26 / 92$ & $02 / 06 / 93$ & F-W & 5 months & 4.58 & 3.36 \\
4 & $09 / 26 / 92$ & $02 / 06 / 93$ & F-W & 5 months & 7.54 & 5.54 \\
5 & $09 / 26 / 92$ & $02 / 06 / 93$ & F-W & 5 months & 3.82 & 2.34 \\
6 & $09 / 26 / 92$ & $02 / 06 / 93$ & F-W & 5 months & 10.68 & 6.19 \\
18 & $02 / 06 / 93$ & $05 / 05 / 93$ & W-Sp & 3 months & 9.64 & 6.52 \\
19 & $02 / 06 / 93$ & $05 / 05 / 93$ & W-Sp & 3 months & 21.84 & 18.36 \\
20 & $02 / 06 / 93$ & $05 / 05 / 93$ & W-Sp & 3 months & 18.88 & 15.88 \\
22 & $02 / 06 / 93$ & $05 / 02 / 93$ & W-Sp & 3 months & 11.24 & 7.96 \\
23 & $05 / 04 / 93$ & $05 / 08 / 93$ & Sp & 4 days & 233.60 & 206.23 \\
24 & $05 / 04 / 93$ & $05 / 08 / 93$ & Sp & 4 days & 274.66 & 176.11 \\
25 & $05 / 04 / 93$ & $05 / 08 / 93$ & Sp & 4 days & 361.35 & 332.15
\end{tabular}

\section{Inner flat zone}

\begin{tabular}{rrrlrrr}
7 & $03 / 16 / 92$ & $11 / 07 / 92$ & Sp-Su-F & 8 months & 9.14 & 3.89 \\
8 & $03 / 16 / 92$ & $02 / 07 / 93$ & Sp-Su-F-W & 11 months & 6.67 & 4.47 \\
9 & $09 / 26 / 92$ & $02 / 07 / 93$ & F-W & 5 months & 8.54 & 3.50 \\
10 & $09 / 26 / 92$ & $02 / 07 / 93$ & F-W & 5 months & 0.00 & 0.00 \\
11 & $09 / 26 / 92$ & $02 / 07 / 93$ & F-W & 5 months & 0.00 & 0.00 \\
12 & $09 / 26 / 92$ & $02 / 07 / 93$ & F-W & 5 months & 0.00 & 0.00 \\
26 & $05 / 04 / 93$ & $05 / 08 / 93$ & Sp & 4 days & 27.38 & 13.69 \\
27 & $05 / 04 / 93$ & $05 / 08 / 93$ & Sp & 4 days & 15.51 & 8.21 \\
28 & $05 / 04 / 93$ & $05 / 08 / 93$ & Sp & 4 days & 75.74 & 37.41 \\
\hline
\end{tabular}

In the mid-flat area, ray feeding pits were mapped in marked areas over several tidal cycles at different times of the year (see table 2). This determined the rate of appearance and disappearance of these structures. In addition, existing ray pits were measured to determine their average dimensions. Ray pit volume
Aunque muchos animales contribuyen a la bioturbación del sedimento en bahía La Choya, este estudio se concentra en los efectos de dos de los más conspicuos fabricantes de huellas: los camarones calianásidos y las rayas. El camarón crea una red tridimensional de galerías (fig. 1). I as rayas producen depresiones en 


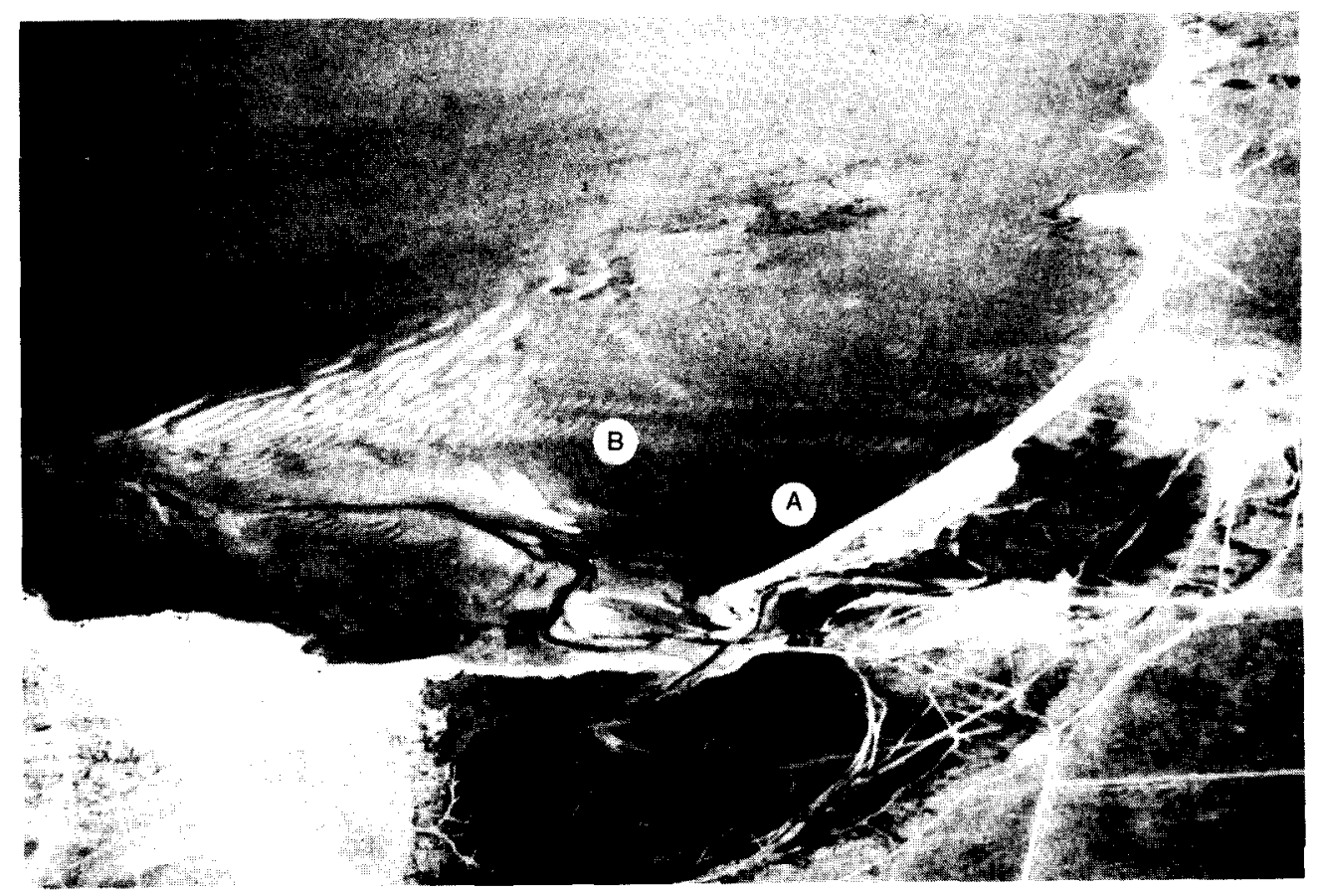

Figure 4. Oblique aerial photograph of Bahia La Choya. A) Location of shell burial experiments. B) Location of ray pit plots.

Figura 4. Fotografia aérea oblicua de bahía La Choya. A) Localización de los experimentos de enterramiento de conchas. B) Localización de los lotes de hoyos de rayas.

was determined by filling in existing pits with known volumes of sand.

\section{Sediment reworking by Callianassa}

Shells of the bivalve mollusk Chione spp. were painted and placed on the surface, concave down, in plots of $0.0625 \mathrm{~m}^{2}$ (fig. 6). The shells used varied in size from approximately 2 to $5 \mathrm{~cm}$. The plots were marked with stakes and left for varying periods of time, from four days to eleven months. These experiments were placed in two areas of the inner flats: one densely populated with the ghost shrimp Callianassa californiensis (the callianassid zone), and the other in which the shrimp is rare or absent (the inner flat zone). Six of the twenty-eight plots could not be relocated (the forma de plato, que ocasionalmente preservan una forma de la raya (fig. 2). Las especies que probablemente son responsables de estas huellas son el cannarón fantasma Callianassa californiensis y las rayas Urolophus halleri y Myliobatis californica (fig. 5). Adicionalmente, el pez guitarra nariz de pala Rhinobatos productus está presente en bahía La Choya y puede contribuir a la producción de hoyos de alimentación. Se observaron todas estas especies en bahía La Choya durante este estudio.

\section{MÉTODOS}

Se siguió el revolvimiento de sedimentos por camarones calianásidos mediante conchas teñidas que se colocaron en la superficie del sedimento en la zona de calianásidos de las pla- 
Table 2. Sediment reworking by bottom-feeding rays. Total pits refers to all feeding traces within the plot; new pits is number first appearing. Reworking rate is calculated as (new pit density) $\times$ (average pit volume $\left.\left[=0.0074 \mathrm{~m}^{3}\right]\right) \times$ (number of high tides per year $[=704]$ ).

Tabla 2. Retrabajamiento de sedimento por rayas alimentadoras del fondo. El total de hoyos (total pits) se refiere a lodas las tracas de alimentación dentro del área y nuevos hoyos (new pits) al númcro que aparecieron por primera vez. La razón de retrabajamiento se calculó como (densidad del hoyo nuevo) $\times\left(\right.$ volumen promedio del hoyo $\left.\left[=0.0074 \mathrm{~m}^{3}\right]\right) \times($ número de pleamares por año $[=704])$.

\begin{tabular}{|c|c|c|c|c|c|c|}
\hline $\begin{array}{l}\text { Date and } \\
\text { time } \\
(\mathrm{m} / \mathrm{d} / \mathrm{yr})\end{array}$ & Season & $\begin{array}{l}\text { Plot and } \\
\text { dimensions } \\
\text { (m) }\end{array}$ & Total pits & New pits & $\begin{array}{l}\text { New pit } \\
\text { density } \\
\left(/ \mathrm{m}^{2}\right)\end{array}$ & $\begin{array}{c}\text { Reworking } \\
\text { rate } \\
\left(\mathrm{m}^{3} / \mathrm{m}^{2} / \mathrm{yr}\right)\end{array}$ \\
\hline 11/07/92 a.m. & Fall & A $10 \times 20$ & 1 & 1 & 0.005 & 0.026 \\
\hline 11/07/92 p.m. & Fall & A $10 \times 20$ & 3 & 2 & 0.010 & 0.052 \\
\hline 11/08/92 a.m. & Fall & A $10 \times 20$ & 24 & 21 & 0.105 & 0.547 \\
\hline 02/06/93 p.m. & Winter & A $10 \times 20$ & 13 & 13 & 0.065 & 0.339 \\
\hline 02/07/93 a.m. & Winter & A $10 \times 20$ & 29 & 16 & 0.080 & 0.417 \\
\hline 04/24/93 a.m. & Spring & A $10 \times 20$ & 151 & 151 & 0.755 & 3.932 \\
\hline 04/25/93 a.m. & Spring & A $10 \times 20$ & 46 & 0 & 0.000 & 0.000 \\
\hline 02/06/93 a.m. & Winter & B $15 \times 20$ & 10 & 10 & 0.033 & 0.172 \\
\hline 02/06/93 p.m. & Winter & B $15 \times 20$ & 4 & 0 & 0.000 & 0.000 \\
\hline 02/07/93 a.m. & Winter & B $15 \times 20$ & 7 & 0 & 0.000 & 0.000 \\
\hline 05/04/93 a.m. & Spring & C $20 \times 30$ & 113 & 113 & 0.188 & 0.979 \\
\hline 05/05/93 a.m. & Spring & C $20 \times 30$ & 9 & 0 & 0.000 & 0.000 \\
\hline 05/06/93 a.m. & Spring & C $20 \times 30$ & 91 & 82 & 0.137 & 0.714 \\
\hline 05/07/93 a.m. & Spring & C $20 \times 30$ & 270 & 179 & 0.298 & 1.552 \\
\hline 05/04/93 a.m. & Spring & D $15 \times 20$ & 229 & 229 & 0.763 & 3.975 \\
\hline 05/05/93 a.m. & Spring & D $15 \times 20$ & 9 & 0 & 0.000 & 0.000 \\
\hline 05/06/93 a.m. & Spring & D $15 \times 20$ & 91 & 82 & 0.274 & 1.427 \\
\hline 05/07/93 a.m. & Spring & D $15 \times 20$ & 329 & 238 & 0.793 & 4.131 \\
\hline
\end{tabular}

stakes had been removed). The relocated plots were carefully excavated, and the depths at which each shell was found recorded. Recovery rates for shells varied from 0 to $92 \%$. Recovery was generally higher for shorter term plots than longer term plots. Recovery was also higher in the callianassid zone than in the inner flat zone.

One tidal cycle's accumulation of sediment is shown in fig. 6 , indicating that burial occurs nicies internas y en las zonas libres de calianásidos de las planicies internas. Para determinar las máximas profundidades de los calianásidos en esta área, se obtuvieron moldes vertiendo resina en las galerías de la zona de calianásidos. Se determinó la densidad promedio de madrigueras por el conteo de las aberturas por metro cuadrado. Las fechas y la duración de los estudios se muestran en la tabla 1. 
A

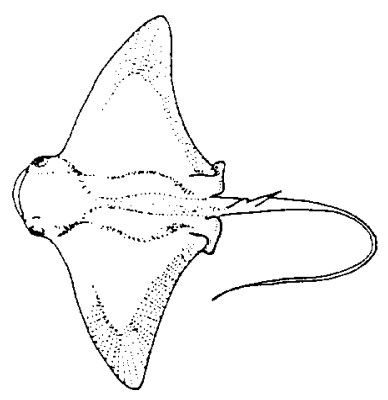

B

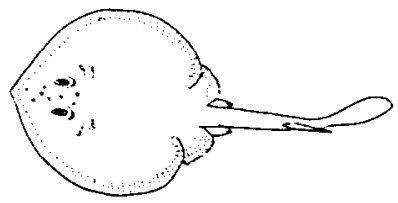

Figure 5. Two rays responsible for the production of ray pits in Bahía La Choya. A) Myliobatis californica (bat ray or raya gavilán), maximum length approximately $120 \mathrm{~cm}$. B) Urolophus halleri (round stingray or raya de espina), maximum length approximately $60 \mathrm{~cm}$. Adapted from Thomson and McKibbin (1976).

Figura 5. Las dos rayas responsables de la producción de los hoyos de raya en bahía La Choya. A) Myliobatis californica (raya murciélago o raya gavilán), con una longitud máxima de approximadamente $120 \mathrm{~cm}$. B) Urolophus halleri (pastinaca redonda o raya de espina), con una longitud máxima de approximadamente $60 \mathrm{~cm}$. Adaptado de Thomson y McKibbin (1976).

almost immediately in the callianassid zone. However, in the area outside the callianassid zone (the inner flat zone), lateral movement of the shells landward is dominant over burial. This is supported by the fact that several of the experiments set up in this area resulted in no shell recovery at all and that shell recovery in the inner flat area was lower overall than in the callianassid zone. In addition, though identical numbers of replicate experiments were set up in the two areas, the rate of loss of experiments was higher in the inner flat area.

The rate of overturn of sediment (accretion rate) for each experimental plot was annualized (see table 1). Maximum rates for each time period were determined using only the depths of those shells actually recovered for all the experiments run for that time interval, normalized to an annual rate. Minimum rates included depths of zero for those shells not recovered for each plot, the assumption being that if the shells were no longer within the area of the plot, they had been removed from the surface and relocated to another area, either on the surface or subsequently buried to an unknown depth. Minimum accretion rates differed markedly from maximum accretion rates in the inner flat zone, where shell recovery averaged only $34 \%$.
En el área de planicie media, los hoyos de alimentación de rayas se trazaron en áreas marcadas durante varios ciclos de marea y épocas del año (tabla 2). Esto determinó la razón de aparición y desaparición de estas estructuras. Adicionalmente, se midieron los hoyos de rayas existentes para determinar sus dimensiones promedio. El volumen de los hoyos fue determinado rellenando las oquedades con volúmenes conocidos de arena.

\section{Retrabajamiento del sedimento por Callianassa}

Conchas del molusco bivalvo Chione spp. fueron pintadas y puestas en la superficie, con la concavidad hacia abajo, en lotes de $0.0625 \mathrm{~m}^{2}$ (fig. 6). Las conchas utilizadas variaron en tamaño de aproximadamente 2 a $5 \mathrm{~cm}$. Se marcaron los lotes con estacas y se dejaron por periodos de tiempo variables, de cuatro días a once meses. Se colocaron estos experimentos en dos áreas de las planicies internas: una densamente poblada con el camarón fantasma Callianassa californiensis (la zona de calianásidos), y otra en la cual el camarón es escaso o ausente (la zona de planicie interna). Seis de los veintiocho lotes no se pudieron relocalizar (se 

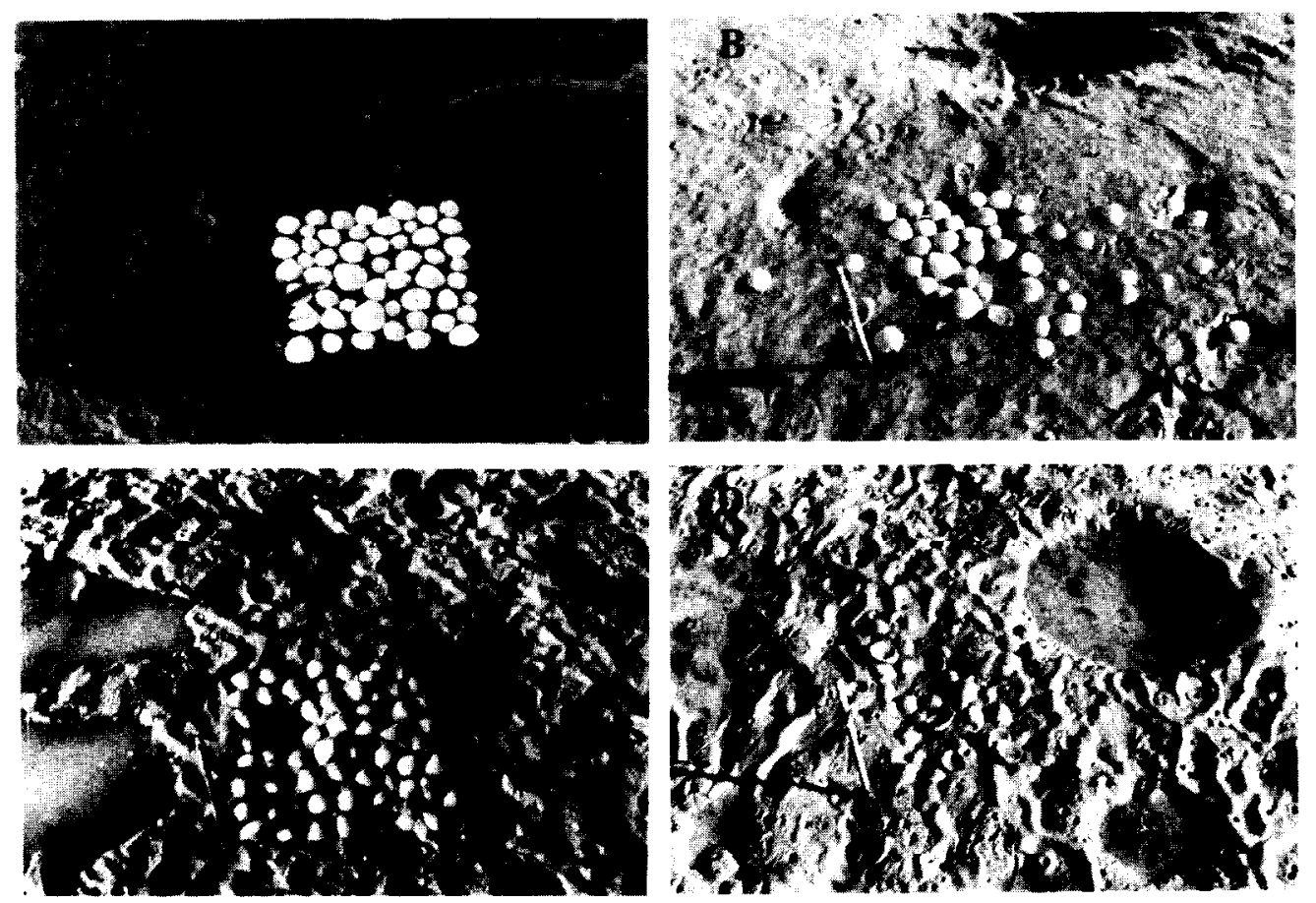

Figure 6. Shell burial experiments from September 1992. a) Inner flat zone experiment as set up. b) Same experiment after one tidal cycle. c) Callianassid zone experiment as set up. d) Same experiment after one tidal cycle. The two depressions in $d$ (upper and lower right) are ray pits.

Figura 6. Experimentos de enterramiento de conchas en septiembre de 1992. a) Experimento montado en la planicie interna. b) El mismo experimento después de un ciclo de marea. c) Experimento montado en la zona calianásida. d) El mismo experimento después de un ciclo de marea. Las dos depresiones en $\mathrm{d}$ (a la derecha superior e inferior) son hoyos de raya.

\section{Rate of production of ray feeding traces}

Four areas were marked in the mid-flat area of the tidal flat (table 2, see fig. 4), all existing ray pits were filled in, and pits appearing after subsequent tides were mapped. See table 2 for a summary of ray pit activity in these experimental plots. This approach enabled a "time-lapse" view of the areas with respect to ray pit accumulation and disappearance. The plots covered both the crests and troughs of sand waves, the dominant physical sedimentary structure in the mid- and outer flats. This corrected for the possibility that the rays preferred to feed in either habian removido las estacas). Se excavaron cuidadosamente los lotes relocalizados y se registró la profundidad a que aparecía cada concha. La razón de recuperación de conchas varió de 0 a $92 \%$. Generalmente, la recuperación fue más alta para los lotes de menor tiempo que para los de mayor tiempo. La recuperación también fue más alta en la zona de calianásidos que en la zona de planicie interna.

En la fig. 6 se muestra la acumulación de sedimento durante un ciclo de marea, indicando que el enterramiento ocurre casi inmedialamente en la zona de calianásidos. Sin embargo, en el área fuera de la zona de calianásidos (la 
troughs or crests. A sample set of these maps is shown in fig. 7. During the spring, when rays become much more abundant in Bahía $\mathrm{La}$ Choya, pits can overlap and cover a large part of the plot's surface area, making differentiation between new and old pits difficult.

\section{RESULTS}

\section{Sediment reworking by Callianassa}

Individual shells in the plots within the callianassid zone were recovered as deep as $17 \mathrm{~cm}$ (within eight months) and $7 \mathrm{~cm}$ (within four days). Shells from the inner flat area were recovered as deep as $12 \mathrm{~cm}$ (in eleven months) and $3 \mathrm{~cm}$ (in four days). Average annualized sediment accretion rates ranged from 2.34 to $361.35 \mathrm{~cm}$ in the callianassid zone, and from 0.00 to $75.74 \mathrm{~cm}$ in the inner flat zone (table 1).

The rate at which the callianassids rework the sediment can be expressed as the difference between the accretion rate in the inner flat zone and the accretion rate in the callianassid zone. Sediment reworking within the inner flat zone could be the result of physical processes, such as ripple migration, or of bioturbation by other organisms. When the area of the sample plots is taken into account $\left(0.0625 \mathrm{~m}^{2}\right)$, reworking rates in the callianassid zone average $0.68 \mathrm{~m}^{3} / \mathrm{m}^{2} /$ year, whereas in the inner flat zone, they average $0.12 \mathrm{~m}^{3} / \mathrm{m}^{2} /$ year. The difference in these rates, which is the reworking due to callianassids, equals $0.56 \mathrm{~m}^{3} / \mathrm{m}^{2} /$ year. The maximum difference in rates is $2.86 \mathrm{~m}^{3} / \mathrm{m}^{2} /$ year, whereas the minimum is $0.02 \mathrm{~m}^{3} / \mathrm{m}^{2} /$ year.

Resin castings of burrows demonstrated that callianassids can burrow to a depth of up to $1.15 \mathrm{~m}$. If this represents the base of the burrowing zone, then reworking the entire thickness of sediment in the Callianassa zone would take an average of two years. Using the maximum observed rate, 0.4 years would be required; using the minimum observed rate, 49 years would be necessary.

The relationship between estimated accretion rates and the period of observation is shown in fig. 8. The longest-term estimate of sedimentation (for the inner flat zone) is provided by a 2,000-year-old shell recovered from zona de planicie interna), el movimiento lateral de las conchas hacia tierra es dominante sobre el enterramiento. Esto está sustentado por el hecho que de varios de los experimentos colocados en esta area no se recuperaron conchas y la recuperación de conchas en el área de la planicie interna fue en general menor que en la zona de calianásidos. Adicionalmente, aunque se colocó un número idéntico de réplicas en los experimentos en las dos áreas, la razón de pérdida de experimentos fue mayor en el área de la planicie interna.

La razón de revolvimiento de sedimento (razón de acreción) para cada lote experimental fue "anualizado" (tabla 1). Se determinó la máxima razón para cada periodo de tiempo usando sólo las profundidades de aquellas conchas que se recuperaron para todos los experimentos que se llevaron a cabo para ese intervalo de tiempo, normalizadas a una razón anual. Las razones minimas incluyeron profundidades de cero para aquellas conchas que no se recuperaron para cada lote, suponiendo que si las conchas ya no estaban dentro del área del lote, habían sido trasladadas de la superficie y relocalizadas en otra área, ya sea en la superficie o subsecuentemente enterradas a una profundidad desconocida. La mínima razón de acreción difirió marcadamente de la máxima razón de acreción en la zona de planicie interna, donde la recuperación de conchas promedió solo $34 \%$.

\section{Razón de producción de huellas de alimentación de rayas}

Se marcaron cuatro áreas en la zona de la planicie media (tabla 2, ver fig. 4), se rellenaron todos los hoyos de rayas existentes, y se trazaron los hoyos que aparecieron en las mareas subsecuentes. La tabla 2 resume la actividad de los hoyos de raya en los experimentos. Este método permite una perspectiva durante un periodo de tiempo con respecto a la acumulación y desaparición de hoyos de rayas. Los lotes cubrieron las crestas y los valles de las ondas de arena, la estructura sedimentaria dominante en las planicies media y externa. Esto fue para corregir la posibilidad de que las rayas prefirieran alimentarse ya sea en los valles o en las 

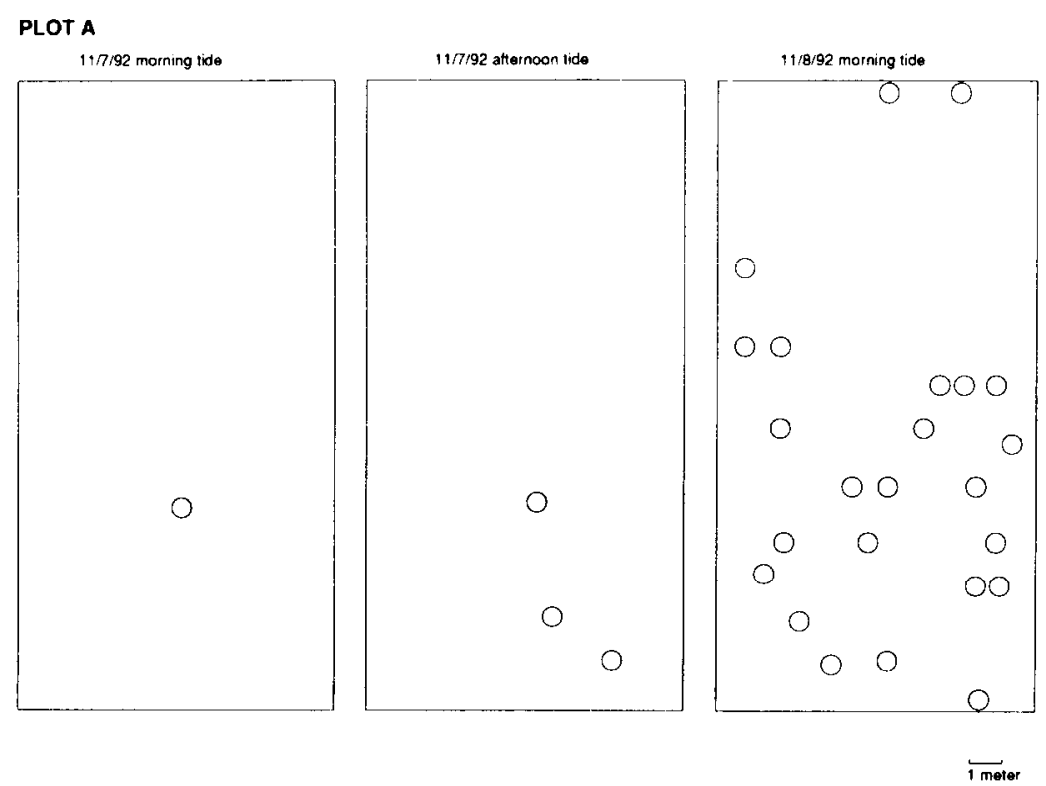

Figure 7. Ray plot A during three consecutive tides. Each circle indicates a single ray pit, slightly larger than actual size.

Figura 7. Lote A de las rayas durante tres mareas consecutivas. Cada círculo indica un solo hoyo de raya, ligeramente más grande que el tamaño real.

a depth of $52 \mathrm{~cm}$ by Flessa et al. (1993). Sadler (1981) documented that estimated accretion rates decrease with respect to the time interval of observation. In Bahía La Choya, this trend demonstrates that even on time scales of days to months, estimates of accretion rates will decrease as the time period of observation increases. Longer-term rates incorporated time periods when sedimentation slowed or ceased, as well as those times in which erosion occurred. This indicates that even the thin $(\sim 1 \mathrm{~m})$ sedimentary sequence in Bahía La Choya is not only intensely bioturbated, but is also incomplete.

\section{Reworking rates due to elasmobranch rays}

The average ray pit in Bahia La Choya is $30 \times 36 \mathrm{~cm}, 6.6 \mathrm{~cm}$ deep and has a volume of $7.41\left(0.0074 \mathrm{~m}^{3}\right)$. However, this appears to be an average for two distinct types of pits. The two species of rays found in Bahia La Choya crestas. Se presentan unas muestras de estos mapas en la fig. 7. Durante la primavera, cuando las rayas se vuelven mucho más abundantes en bahía La Choya, los hoyos pueden traslaparse y cubrir una gran parte de los lotes en el área superficial; esto dificulta la distinción entre hoyos nuevos y viejos.

\section{RESULTADOS}

\section{Retrabajamiento de sedimento por Callianassa}

Se recuperaron las conchas individuales en los lotes dentro de la zona de calianásidos hasta una profundidad de $17 \mathrm{~cm}$ (dentro de ocho meses) y $7 \mathrm{~cm}$ (dentro de cuatro días). Las conchas en el área de planicie interna se recuperaron hasta una profundidad de $12 \mathrm{~cm}$ (en once meses) y $3 \mathrm{~cm}$ (en cuatro dias). La acreción promedio anualizada de sedimento osciló de 2.34 a $361.35 \mathrm{~cm}$ en la zona de calianásidos y 

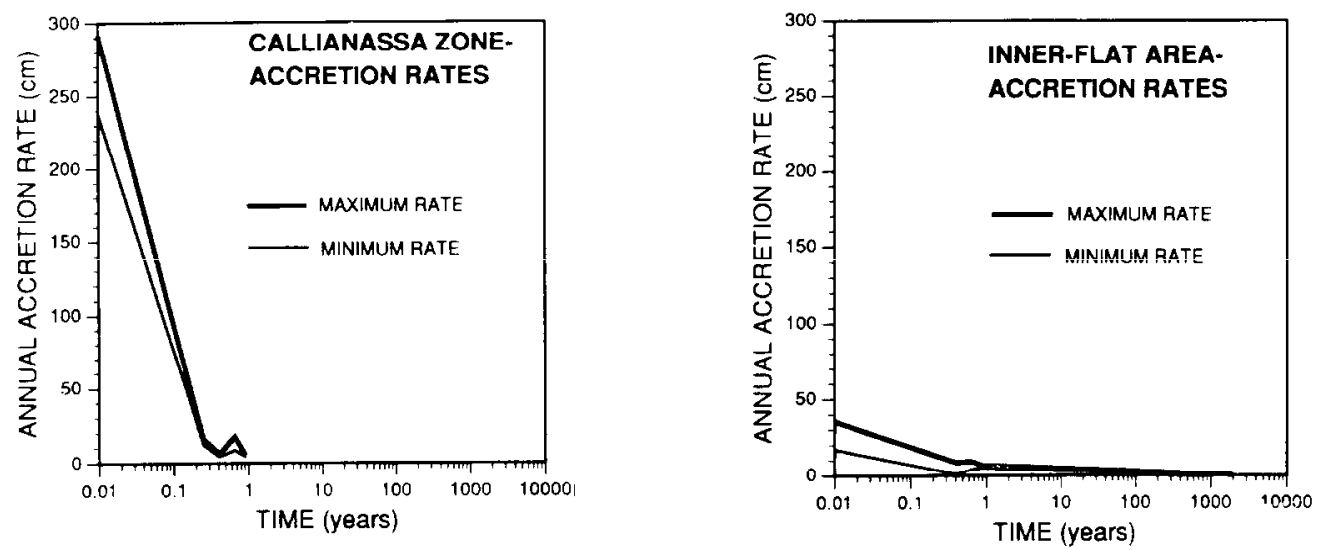

Figure 8. Accretion rates for shell burial experiments. Each point is the combined mean for all experiments run for the length of time indicated on the $x$-axis (see table 1). The 2,000 year point on the inner flat area graph is the rate estimated by Flessa et al. (1993).

Figura 8. Razones de acreción para los experimentos de enterramiento de conchas. Cada punto es la media combinada de todos los experimentos hechos durante el tiempo indicado en el eje $x$ (ver tabla 1). El punto que representa 2,000 años en la gráfica de la planicie interna es la razón estimada por Flessa et al. (1993).

differ in size and shape; the elliptical bat rays can reach twice the size of the round stingrays. The end result is two "populations" of ray pits: one type of ray pit (made by bat rays) is large and ellipsoidal, whereas the other type is small and almost circular (made by round stingrays) The combined distribution of these two populations of ray pits is shown in fig. 9.

Smaller pits are much more common than the larger pits (fig. 10): either the smaller rays (round stingrays) are more common than the larger rays (bat rays), or smaller rays produce pits more frequently than larger rays.

Table 2 shows that the number of now pits in a particular area in Bahia La Choya is highly variable, and can increase or decrease tenfold within a day. This indicates that these structures have a high disappearance, or infilling, rate (see Grant, 1983, for discussion of the process of infilling of ray pits). The data also suggest that reworking rates are higher in the spring than in the other seasons sampled.

Given that the average ray pit has a volume of $0.0074 \mathrm{~m}^{3}$, the average yearly reworking rate in Bahia La Choya is $1.01 \mathrm{~m}^{3} / \mathrm{m}^{2} /$ year (see de 0.00 a $75.74 \mathrm{~cm}$ en la zona de planicie interna (tabla 1$)$

La razón a la cual los calianásidos retrabajan los sedimentos puede expresarse como la diferencia entre la razón de acreción en la zona de planicie interna y la razón de acreción en la zona de calianásidos. El retrabajamiento de sedimento dentro de la zona de planicie interna puede ser el resultado de procesos físicos, tales como la migración de rizaduras o la bioturbación por otros organismos. Cuando se toma en cuenta el área de los lotes de muestra $\left(0.0625 \mathrm{~m}^{2}\right)$, las razones de retrabajamiento son, en promedio, $0.68 \mathrm{~m}^{3} / \mathrm{m}^{2} /$ año en la zona de calianásidos y $0.12 \mathrm{~m}^{3} / \mathrm{m}^{2} /$ año en la zona de planicie interna. La máxima diferencia en las razones es $2.86 \mathrm{~m}^{3} / \mathrm{m}^{2} / a n ̃ o$, mientras que el mínimo es $0.02 \mathrm{~m}^{3} / \mathrm{m}^{2} /$ año.

Los moldes de resina de las madrigueras demostraron que los calianásidos pueden enterrarse hasta una profundidad de $1.15 \mathrm{~m}$. Si esto representa la base de la zona de enterramiento, entonces el retrabajamiento del espesor total de sedimento en la zona de calianásidos tomaría un promedio de dos años. Usando la razón 
table 2). Because the rays can excavate sediment to a depth of $20 \mathrm{~cm}$, approximately 72 days would be required for the rays to overturn the sediment in Bahía La Choya to the base of their known burrowing zone. This is an average value, however, calculated with data from three seasons. The maximum rate of reworking, observed on May 7,1993 , is $4.13 \mathrm{~m}^{3} / \mathrm{m}^{2} /$ year. This rate would require only 17 days for the complete reworking of the upper $20 \mathrm{~cm}$ of sediment.

\section{IMPLICATIONS FOR THE SEDIMENTARY RECORD}

Both callianassid shrimp and elasmobranch rays have a profound effect on the sediments of Bahía La Choya. Both can rework large amounts of sediment in a short time.

The high sediment overturn rates caused by these organisms are likely to destroy most small-scale sedimentary structures. For example, the abundant ripple marks of the outer flats (see Fürsich and Flessa, 1987) are not likely to be preserved in the fossil record. Nor are many of the other biogenic structures of these tidal flats likely to withstand the effects of the shrimp and rays. Larger sedimentary structures, such as the sand waves of the outer flats, are more likely to be preserved. Their relief (approximately $50 \mathrm{~cm}$ ) greatly exceeds the maximum depth of ray reworking. Smaller sedimentary structures, though much more abundant at any instant in time, will not be as well represented in the sedimentary record. Only a few may escape the homogenizing effects of ray and shrimp bioturbation. Rare episodes of rapid and deep burial might preserve ripple marks and small-scale biogenic structures below the reach of burrowers. Such burial might occur in this region as a result of the migration of alluvial fans from the nearby headlands or by the migration of the adjacent beach.

Although actualistic studies are important in understanding the physical and biological processes that can affect sedimentary deposits, the short time frame of their observations can be misleading. This study suggests that the vast expanses of rippled tidal flats in this low sedimentation rate region are ephemeral features, máxima observada, se requeriría de 0.4 años; usando la razón mínima observada, serían necesarios 49 años.

La interrelación entre las razones de acreción estimadas y el periodo de observación se muestra en la fig. 8. La estimación de la razón de sedimentación a largo plazo (para la zona de planicie interna) es proporcionada por una concha de 2,000 años de edad, que se recuperó de una profundidad de $52 \mathrm{~cm}$ por Flessa et al. (1993). Sadler (1981) documentó esa razón de acreción estimada con respecto al intervalo de tiempo de observación. En bahía La Choya, esta tendencia demuestra que, aun en escalas de tiempo de días a meses, la estimación de las razones de acreción decrecerá conforme se incrementa el periodo de tiempo de las observaciones. Las razones a mayor plazo incorporaron periodos cuando la sedimentación disminuyó o cesó, así como aquellos tiempos en que ocurrió erosión. Esto indica que hasta la secuencia sedimentaria delgada $(\sim 1 \mathrm{~m})$ de bahía La Choya no sólo está intensamente bioturbada, sino también incompleta.

\section{Razones de retrabajamiento debido a rayas}

El hoyo de raya promedio en bahía La Choya es de $30 \times 36 \mathrm{~cm}$, con una profundidad de $6.6 \mathrm{~cm}$ y un volumen de $7.41\left(0.0074 \mathrm{~m}^{3}\right)$. Sin embargo, esto parece ser el promedio de dos distintos tipos de hoyos. Las dos especies de rayas que se encontraron en bahía La Choya difieren en tamaño y forma; las rayas murciélago elipticas pueden ser el doble de tamaño que las pastinacas redondeadas. El resultado final es dos "poblaciones" de hoyos de rayas: un tipo de hoyo de raya (hecho por las rayas murciélago) es grande y elipsoidal, mientras que el otro tipo es pequeño y casi circular (hecho por las pastinacas redondeadas). La distribución combinada de estas dos poblaciones de hoyos de rayas se muestra en la fig. 9.

Los hoyos pequeños son mucho más comunes que los grandes (fig. 10), ya sea porque las rayas más pequeñas (pastinacas) son más comunes que las grandes (rayas murciélago) o porque las rayas más pequeñas producen hoyos más frecuentemente que las rayas más grandes. 


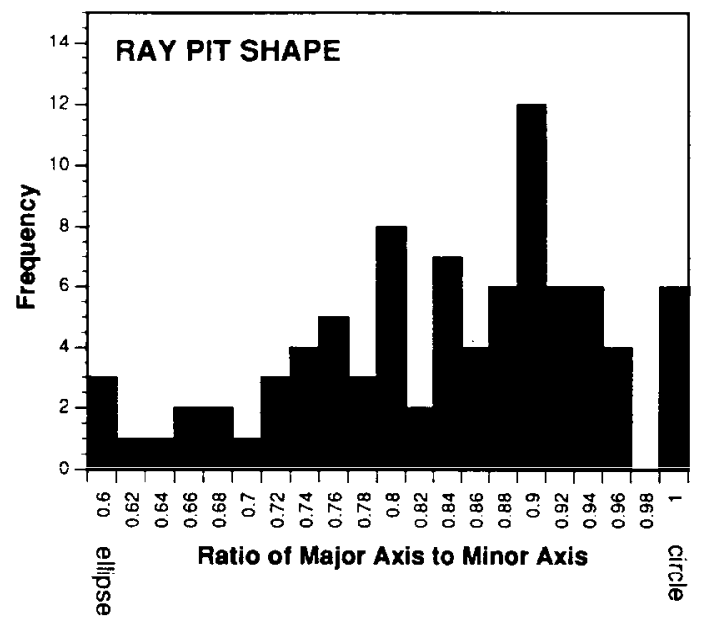

Figure 9. Frequency distribution of ray pit shape. Ellipsoidal pits are produced by bat rays, while circular pits are produced by round stingrays.

Figura 9. Distribución de frecuencia de la forma de hoyo de raya. Las rayas murciélago producen los hoyos elipsoidales, mientras que las pastinacas redondas producen los hoyos circulares.

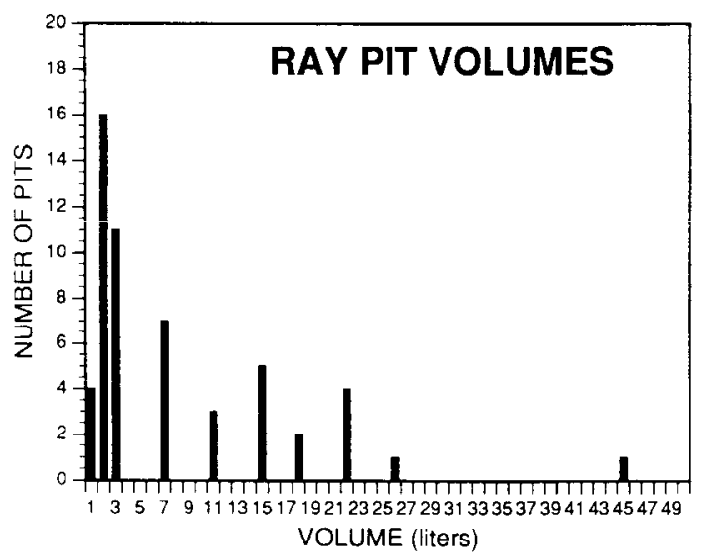

Figure 10. Ray pit volumes in Bahía La Choya. Gaps result from limitations in the measuring technique (filling with known volumes of sand) as well as the natural distribution of ray pit volumes.

Figura 10. Volumen por hoyo de raya en bahía La Choya. Los huecos resultan de las limitaciones en la técnica de medición (llenando con volumen conocido de arena) así como de la distribución natural de los volúmenes de los hoyos de raya. 
unlikely to be preserved in the record. Actualistic studies will have greater utility if the cumulative effects of sediment reworking rates are carefully considered.

Meldahl (1987) suggested that shell beds can form below the sediment surface if the rate of biogenic reworking exceeds the rate of sediment accumulation. His model of biogenic sedimentation was based on the presence of subsurface shell beds in areas of conveyor belt deposit feeders (such as the callianassid shrimp) in Bahía La Choya. Although the circumstantial evidence supported this model, Meldahl did not conduct any controlled experiments to document the nature and rate of the process. This study shows that Meldahl's model is a plausible mechanism for the formation of some shell beds. Shells within the zone of callianassid reworking are, in fact, buried much more quickly than shells outside the shrimp habitat. Reworking rates appear to be much higher than sediment accumulation rates.

Sadler (1981) surveyed the published literature on sedimentation rates and plotted the observed sedimentation rate against the time period used to estimate the rate. He found that sediment accumulation rates decrease as the time span of observation increases. The very high rates of sediment accumulation sometimes observed over brief periods are rarely, if ever, sustained for long periods of time. Longer spans of time are simply more likely to include intervals during which no sediment was added to the deposit, or intervals of time during which sediment was removed. This negative correlation between rate and interval also occurs in the sediments of Bahía La Choya (fig. 8), where physical sedimentation rates are low and the mode of sediment accumulation is largely biogenic. This study documents that even relatively thin $(20-100 \mathrm{~cm})$ sections of intertidal sediments are likely to be incomplete at time scales of weeks to months. Such intervals may not only be incomplete, they are likely to be stratigraphically disordered. Biogenic reworking is likely to disrupt the original superposition of sedimentary particles at potential fossils (see Cutler, 1993, and Sadler, 1993, for further discussion of this point). In one sense, the sedimentary record of Bahia La Choya is a record
La tabla 2 muestra que el número de nuevos hoyos en un área particular en bahia La Choya es altamente variable, y puede incrementarse o decrecer hasta diez veces en un día. Esto indica que estas estructuras tienen una alta razón de desaparición o relleno (ver Grant, 1983, para una discusión del proceso de relleno de los hoyos de rayas). Los datos también sugieren que las razones de retrabajamiento son más altas en la primavera que en las otras estaciones muestreadas.

Dado que el hoyo promedio de raya tiene un volumen de $0.0074 \mathrm{~m}^{3}$, la razón de retrabajamiento anual promedio en bahía La Choya es de $1.01 \mathrm{~m}^{3} / \mathrm{m}^{2} /$ año (ver tabla 2). Debido a que las rayas pueden excavar el sedimento hasta una profundidad de $20 \mathrm{~cm}$, las rayas requieren de aproximadamente 72 días para revolver el sedimento hasta la base de su zona de enterramiento conocida en bahía La Choya. Sin embargo, éste es un valor promedio calculado con datos de tres estaciones. La máxima razón de retrabajamiento, observada el 7 de mayo de 1993, es de $4.13 \mathrm{~m}^{3} / \mathrm{m}^{2} /$ año. Esta razón requiere de solo 17 dias para completar el retrabajamiento de los $20 \mathrm{~cm}$ superiores de sedimento.

\section{IMPLICACIONES PARA EL REGISTRO SEDIMENTARIO}

Tanto los camarones calianásidos como las rayas tienen un profundo efecto sobre los sedimentos de bahía La Choya. Ambos pueden retrabajar grandes cantidades de sedimento en un corto tiempo.

Las altas razoncs de mezclado del sedimento causadas por estos organismos probablemente destruyen la mayoría de las estructuras sedimentarias de pequeña escala. Por ejemplo, es probable que las abundantes rizaduras de las planicies externas (ver Fürsich y Flessa, 1987) no se preserven en el registro fósil, ni que muchas de las estructuras biogénicas de las planicies de marea puedan resistir los efectos de los camarones y las rayas. Es más probable que se preserven las estructuras sedimentarias mayores, tales como las ondas de arena de las planicies externas. Su relieve (de aproximadamente $50 \mathrm{~cm}$ ) excede considerablemente la máxima profundidad de retrabajamiento de las 
of bioturbation. This record's incompleteness is a consequence of bioturbation because sediment accumulation at the surface can only occur if sediment is removed from the sediment at depth and then placed on the surface.

\section{ACKNOWLEDGEMENTS}

Field work was supported by a Grant-inAid from Sigma Xi, NSF grant EAR-89113442, and NATO grant CRG 920691. We thank Sally Walker for her assistance throughout this project and for her comments on earlier versions of this manuscript. Reviews by Mary Droser and Keith Meldahl also improved the manuscript. We also thank Murray Gregory for providing information on ray burrows and Warren Garrett, Tina Wells, Diana Hallman and Michal Kowalewski for field assistance. We thank Miguel Téllez Duarte for the Spanish translation. This is publication number 10 of CEAM (Centro de Estudio de Almejas Muertas).

\section{REFERENCES}

Aller, R.C. and Dodge, R.E. (1974). Animalsediment relations in a tropical lagoon, Discovery Bay, Jamaica. J. Mar. Res., 32: 209-232.

Anderson, A.M. (1976). Fish trails from the Early Permian of South Africa. Palaeontology, 19: 397-409.

Bigelow, H.B. and Schroeder, W.C. (1953). Fishes of the North Atlantic. Part 2. Sawfishes, guitarfish, skates and rays. Sears Foundation for Marine Research, Yale Univ., New Haven, 588 pp.

Bosworth, W.S. and Thibodeaux, L.J. (1990). Bioturbation: a facilitator of contaminant transport in bed sediment. Environ. Prog., 9: 211-217.

Bromley, R.G. (1990). Trace Fossils. Biology and Taphonomy. Unwin Hyman, London, $280 \mathrm{pp}$.

Brook, F.J. (1983). Lower Miocene geology of North Kaipara, northern New Zealand, with emphasis on the paleontology. Ph.D. dissertation, Univ, of Auckland, Auckland, New Zealand, 390 pp. rayas. Las estructuras sedimentarias menores, a pesar de ser mucho más abundantes en cualquier instante de tiempo, serán menos representadas en el registro sedimentario. Sólo unas pocas se escapan del efecto homogeneizador de la bioturbacion de rayas y camarones. Episodios raros de enterramiento rápido y profundo pueden preservar rizaduras y estructuras biogénicas de pequeña escala por debajo del alcance de los enterradores. Tal enterramiento puede ocurrir en esta región como resultado de la migración de los abanicos aluviales cercanos o la migración de la playa adyacente.

No obstante que los estudios "actualísticos" son importantes para entender los procesos físicos y biológicos que pueden afectar los depósitos sedimentarios, el corto tiempo de las observaciones puede conducir a interpretaciones erróneas. Este estudio sugiere que las vastas extensiones de planicies de marea con rizaduras en esta región de baja tasa de sedimentación son características efímeras, y es improbable que sean preservadas en el registro. Estudios "actualísticos" tendrán mayor utilidad si los efectos acumulativos de las razones de retrabajamiento de los sedimentos son cuidadosamente considerados.

Meldahl (1987) sugiere que se pueden formar capas de conchas debajo de la superficie del sedimento si la razón de retrabajamiento biogénico excede la razón de acumulación de sedimento. Su modelo de sedimentación biogénica estuvo basado en la presencia de capas de conchas subsuperficiales en áreas de bandas de sedimentivoros (tales como los camarones calianásidos) en bahía La Choya. A pesar de la evidencia circunstancial que apoyó este modelo, Meldahl no llevó a cabo experimentos controlados para documentar la naturaleza y razón del proceso. Este estudio muestra que el modelo de Meldahl es un mecanismo plausible para la formación de algunas capas de conchas. Las conchas dentro de la zona de retrabajamiento de calianásidos son, de hecho, enterradas mucho más rápido que las conchas fuera del hábitat del camarón. Las razoncs de retrabajamiento parecen ser mucho más altas que las razones de acumulación de sedimentos. 
Clifton, H.E. and Hunter, R.E. (1973). Bioturbational rates and effects in carbonate sand, St. John, U.S. Virgin Islands. J. Geol., 81: 253-268.

Cook, D.O. (1971) Depressions in shallow marine sediment made by benthic fish: $\mathbf{J}$. Sedimen. Petrol., 41: 577-602.

Cutler, A.H. (1993). Mathematical models of temporal mixing in the fossil record. In: S.M. Kidwell and A.K. Behrensmeyer (eds.), Taphonomic Approaches to Time Resolution in Fossil Assemblages. Paleontological Soc. Short Courses in Paleontology No. 6, Univ. of Tennessee, Knoxville, Tennessee, pp. 169-187.

Cutler, A. and Flessa, K.W. (1990). Fossils out of sequence: Computer simulations and strategies for dealing with stratigraphic disorder. Palaios, 5: 227-235.

Droser, M.L. and Bottjer, D.J. (1986). A semiquantitative classification of ichnofabric. $\mathbf{J}$. Sedimen. Petrol., 56: 558-569.

Dubiel, R.F., Blodgett, R.H. and Bown, T.M. (1987). Lungfish burrows in the Upper Triassic Chinle and Dolores Formations, Colorado Plateau. J. Sedimen. Petrol., 57: 512-521.

Ekdale, A.A. (1987). Late Cenozoic rocks in the Puerto Peñasco area. In: K.W. Flessa (ed.), Paleoecology and Taphonomy of Recent to Pleistocene Intertidal Deposits, Gulf of California. Paleontological Soc. Spec. Publ. No. 2, Univ. of Tennessee, Knoxville, Tennessee, pp. 34-43.

Feibel, C.S. (1987). Fossil fish nests from the Koobi Fora Formation (Plio-Pleistocene) of northern Kenya. J. Paleontol., 61: 130-134.

Flessa, K.W., Cutler, A.H. and Meldahl, K.H. (1993). Time and taphonomy: Quantitative estimates of time-averaging and stratigraphic disorder in a shallow marine environment. Paleobiology, 19: 266-286.

Fürsich, F.T. and Flessa, K.W. (1987). Taphonomy of tidal flat molluscs in the northern Gulf of California: Paleoenvironmental analysis despite the perils of preservation. Palaios, 2: 543-559.

Fürsich, F.T., Flessa, K.W., Aberhan, M., Feige, A. and Schödlebauer, S. (1991). Sedimentary habitats and molluscan faunas
Sadler (1981), en una revisión de la literatura publicada sobre razones de sedimentación, graficó la razón observada de sedimentación contra el periodo de tiempo utilizado para estimar la razón. Encontró que la razón de acumulación de sedimento decrece conforme se incrementa el intervalo de tiempo de observación. Raramente, si es que nunca, se mantienen por periodos largos de tiempo las muy altas razones de acumulación de sedimentos que algunas veces se observan. Es más probable que lapsos largos de tiempo incluyan intervalos durante los cuales no se agregó sedimento al depósito, o intervalos de tiempo durante el cual el sedimento fue removido. Esta correlación negativa entre razón e intervalos también ocurre en los sedimentos de bahía La Choya (fig. 8), donde las razones de sedimentación son bajas y el modo de acumulación de sedimentos es mayormente biogénico. Este estudio documenta que aun secciones relativamente delgadas (20-100 cm) de sedimentos intermareales probablemente sean incompletos a escalas de tiempo de semanas a meses. Tales intervalos pueden ser no sólo incompletos, sino muy probablemente estratigráficamente desordenados. Es probable que el retrabajamiento biogénico trastorne la superposición original de las partículas sedimentarias a fósiles potenciales (ver Cutler, 1993, y Sadler, 1993, para una discusión adicional de este punto). En un sentido, el registro sedimentario de bahía La Choya es un registro de bioturbación. Lo incompleto del registro es una consecuencia de la bioturbación debido a que la acumulación de sedimento en la superficie sólo puede ocurrir si el sedimento es removido del mismo a profundidad y colocado sobre la superficie.

\section{AGRADECIMIENTOS}

El trabajo de campo fue financiado por un Grant-in-Aid de Sigma Xi, la NSF (convenio EAR-89113442) y la OTAN (convenio CRG 920691). Agradecemos a Sally Walker su ayuda durante este proyecto y sus comentarios en las primeras versiones del manuscrito. Las revisiones de Mary Droser y Keith Meldahl también ayudaron a mejorar el manuscrito. 
of Bahía La Choya (Gulf of Mexico, Sonora, Mexico). Zitteliana, 18: 5-51.

Grant, J. (1983). The relative magnitude of biological and physical sediment reworking in an intertidal community. J. Mar. Res., 41: 673-689.

Gregory, M.R. (1981). A Miocene analogue of modern eagle ray feeding hollows. Annual Meeting, Geol. Soc. of New Zealand (abstract)

Gregory, M.R. (1986). Trace fossils from basal Waitemata rocks: Goat Island and Goat Island Bay, the Outpost and Cape Rodney, and Matheson's Bay, Leigh District. In: A.E. Wright. and R.E. Beever (eds.), The Offshore Islands of Northern New Zealand. Dept. of Lands and Survey, Information Ser., 16: 42.

Gregory, M.R. (1991). New trace fossils from the Miocene of Northland, New Zealand: Rorschachichnus amoeba and Piscichnus waitemata. Ichnos, 1: 195-205.

Gregory, M.R., Ballance, P.F., Gibson, G.W. and Ayling, A.M. (1979). On how some rays (Elasmobranchia) excavate feeding depressions by jetting water. J. Sedimen. Petrol., 49: 1125-1130.

Gregory, M.R., Ballance, P.F. and Gibson, G.W. (1983). Fossil analogues of modern ray feeding hollows-further examples and additional comments. Annual Meeting, Geol. Soc. of New Zealand (abstract).

Griffis, R.B., and Chavez, F.L. (1988). Effects of sediment type on burrows of Callianassa californiensis Dana and C. gigas Dana. J. Exp. Mar. Biol. Ecol., 117: 239-253.

Howard, J.D., Mayou, T.V. and Heard, R.W. (1977). Biogenic sedimentary structures formed by rays. J. Sedimen. Petrol., 47: 339-346.

Hunter, R.E., Clifton, H.E., Hall, N.T. and Chin, J.L. (1984). Pleistocene shoreline and shelf deposits at Fort Funston and their relation to sea-level changes. SEPM, 1984 Midyear Meeting, San Jose, California, Field Trip Guidebook, 3: 1-30.

Kamola, D.L. (1984). Trace fossils from marginal-marine facies of the Spring Canyon Member, Blackhawk Formation (Upper Cretaceous), East-Central Utah. J. Paleontol., 58: 529-541.
Agradecemos a Murray Gregory su información sobre los hoyos de rayas y a Warren Garrett, Tina Wells, Diana Hallman y Michal Kowalewski su ayuda en el campo. Ésta es la publicación número 10 del CEAM (Centro de Estudios de Almejas Muertas).

Finalmente, agradecemos a Miguel Téllez Duarte la traducción al español.

MacGinitie, G.E. (1935). Ecological aspects of a California marine estuary. American Midland Naturalist, 16: 629-765.

MacGinitie, G.E. and MacGinitie, N. (1949). Natural History of Marine Animals. McGraw-Hill, New York, 473 pp.

McAllister, J.A. (1988). Lungfish burrows in the Upper Triassic Chinle and Dolores Formations, Colorado Plateau: Comments on the recognition criteria of fossil lungfish burrows. J. Sedimen. Petrol., 58: 365-369.

Meldahl, K.H. (1987). Sedimentologic, stratigraphic, and taphonomic implications of biogenic stratification. Palaios, 2: 350-358.

Meyers, A.C. (1977a). Sediment processing in a marine subtidal sandy bottom community: I. Physical aspects. J. Mar. Res., 35: 609-632.

Mcycrs, A.C. (1977b). Sediment processing in a marine subtidal sandy bottom community: II. Biological consequences. J. Mar. Res., 35: 633-647.

Miller, M.F. (1979). Paleoenvironmental distribution of trace fossils in the Catskill deltaic complex, New York State. Palaeogeogr., Palaeoclimatol., Palaeoecol., 28: 117-141.

Miller, M.F. and Myrick, J.L. (1992). Population fluctuations and distributional controls of Callianassa californiensis: Effect on the sedimentary record. Palaios, 7: 621-625.

Miller, M.F., Curran, H.A. and Myrick, J.L. (1990). A test for taphonomic inhibition: Do shell layers inhibit the burrowing of callianassid shrimp? Geol. Soc. of America Abstracts with Programs, 22: 83.

Nichols, M.N. (1965). Composition and environment of Recent transitional sediments of the Sonoran coast of Mexico. Ph.D. dissertation, Univ. of California, Los Angeles, $401 \mathrm{pp}$. 
Nordell, S.E. (1990). The courtship and mating behavior of the round stingray, Urolophus halleri. M.Sc. thesis, Univ. of Arizona, Tucson, $47 \mathrm{pp}$.

Ott, J.A., Fuchs, B., Fuchs, R. and Malasek, A. (1976). Observations on the biology of Callianassa stebbingi Borrodaille and Upogebia litoralis Risso and their effect on the sediment. Senckenbergiana Maritima, 8: 61-79.

Over, D.J. (1990). Trace metals in burrow walls and sediments, Georgia Bight, USA. Ichnos, 1 : 31-41.

Pemberton, J.S., Risk, M.J. and Buckley, D.E. (1976). Supershrimp: deep bioturbation in the Strait of Canso, Nova Scotia. Science, 192: 709-791.

Posey, M.H. (1986). Changes in a benthic community associated with dense beds of a burrowing deposit feeder, Callianassa californiensis. Mar. Ecol. Prog. Ser., 31: 15-22.

Risk, M.J. and Craig, H.D. (1976). Flatfish feeding traces in the Minas basin. J. Sedimen. Petrol., 46: 411-413.

Ronan, T.E., Jr, Miller, M.F. and Farmer, J.D. (1981). Organism-sediment relationships on a modern tidal flat, Bodega Harbor, California. In: V. Frizzell (ed.), Modern and Ancient Biogenic Structures. Annual Meeting Pacific Section SEPM, Field Trip 3 Guide, pp. 15-31.

Sadler, P.M. (1981) Sediment accumulation rates and the completeness of stratigraphic sections. J. Geol., 89: 569-584.

Sadler, P.M. (1993). Models of time-averaging as a maturation process: How soon do sedimentary sections escape reworking? In: S.M. Kidwell and A.K. Behrensmeyer (eds.), Taphonomic Approaches to Time Resolution in Fossil Assemblages. Paleontological Soc. Short Courses in Paleontology No. 6, Univ. of Tennessee, Knoxville, Tennessee, pp. 188-209.

Shinn, E.A. (1968). Burrowing in Recent lime sediments of Florida and the Bahamas. J. Paleontol., 42: 879-894.

Stanley, D.J. (1971). Fish-produced markings on the outer continental margin east of the Middle Atlantic States. J. Sedimen. Petrol., 41: 159-170.
Suchanek, T.H. (1983). Control of seagrass communities and sediment distribution by Callianassa (Crustacea, Thalassinidea) bioturbation. J. Mar. Res., 41: 281-298.

Swinbanks, D.D. and Luternauer, J.L. (1987). Burrow distribution of thalassinidean shrimp on a Fraser Delta tidal flat, British Columbia. J. Paleontol., 61: 315-332.

Tamaki, A. (1988). Effects of the bioturbating activity of the ghost shrimp Callianassa japonica Ortmann on migration of a mobile polychaete. J. Exp. Mar. Biol. Ecol., 120: 81-95.

Taylor, A.M. and Goldring, R. (1993). Description and analysis of bioturbation and ichnofabric. J. Geol. Soc., London, 150 : 141-148.

Thomson, D.A. and McKibben, N. (1976). Gulf of California Fishwatcher's Guide. Golden Puffer Press, Tucson, Arizona, $75 \mathrm{pp}$.

Thrush, S.F., Pridmore, R.D., Hewitt, J.E. and Cummings, V.J. (1991). Impact of ray feeding disturbances on sandflat macrobenthos: do communities dominated by polychaetes or shellfish respond differently? Mar. Ecol. Prog. Ser., 69: 245-252.

Tudhope, A.W. and Scoffin, T.P. (1984). The effects of Callianassa bioturbation on the preservation of carbonate grains in Davies Reef lagoon, Great Barrier Reef, Australia. J. Sedimen. Petrol., 54: 1091-1096.

Vaugelas, J.V. de (1984). Preliminary observations on two types of callianassid (Crustacea, Thalassinidea) burrows, Gulf of Aqaba (Red Sea). In: M.A.H. Saad (ed.), Proc. of the Symposium on Coral Reef Environments of the Red Sea. King Abdulaziz Univ. Press, Jeddah, pp. 520-539.

Warme, J.E. (1967). Graded bedding in the Recent sediments of Mugu Lagoon, California. J. Sedimen. Petrol., 37: 540-547.

Warme, J.E. (1971). Paleoecological aspects of a modern coastal lagoon. Univ. California Publ. Geol. Sci., 87: 1-110.

Whitky, G.P. (1940). The Fishes of Australia. Part 1. The sharks, rays, devil fish, and other primitive fish of Australia and New Zealand. Royal Zool. Soc. of New South Wales, Sydney, pp. 1-280. 